\title{
Easy does it: The role of fluency in cue weighting
}

\author{
Anuj K. Shah* and Daniel M. Oppenheimer \\ Princeton University
}

\begin{abstract}
We propose that people weight fluent, or easy to process, information more heavily than disfluent information when making judgments. Cue fluency was manipulated independent of objective cue validity in three studies, the findings from which support our hypothesis. In Experiment 1, participants weighted a consumer review more heavily when it was written in a clear font than in a less clear font. In Experiment 2, participants placed more weight on information when it was in focus than when it was blurry. In Experiment 3, participants placed more weight on financial information from brokerage firms with easy to pronounce names than those with hard to pronounce names. These studies demonstrate that fluency affects cue weighting independent of objective cue validity.
\end{abstract}

Keywords: fluency, cue weighting, judgment.

\section{Introduction}

When making judgments and decisions, people have numerous pieces of information (i.e., cues) available to aid in the decision process. When deciding whether a patient has diabetes, a doctor might examine the patient's symptoms, family health history, or lab results. A stockbroker might consider companies' profit margins, quarterly projections, and recent activity. A judge or jurist might consider testimony from several witnesses and various other pieces of evidence. With so much information available, how do we decide which cues to weight most heavily?

Conventional models suggest that decision-makers should weight information based on objective cue validity. According to normative principles such as the weighted additive (Payne, Bettman, \& Johnson, 1993), additive difference (Tversky, 1969), and weighted averaging models (Himmelfarb, 1970), people should consider all of the available cues that might inform a particular judgment. They should then differentially weight each cue based upon how successfully the cue predicts the outcome. However, this approach cannot perfectly explain how naïve decision-makers, who are unaware of cue validities, might make judgments or decisions. Nor can it explain how people's judgments often result from overweighting less valid information (Tversky \& Kahneman,

\footnotetext{
*The research was supported in part by Grant \# 0518811 from the National Science Foundation. We would like to thank Sara Etchison, Gina Mamikonyan, and Melissa Miller for assisting with data collection, Sunny Khemlani for his help in designing stimuli, and Jonathan Baron, Reed Hunt, Rolf Reber, Ed Wisniewski, several anonymous reviewers, and the entire Oppenheimer Lab for their extensive feedback on this work. Address: Anuj K. Shah, Princeton University, Dept. of Psychology,Green Hall, Princeton, NJ 08544. Email: akshaheprinceton. edu
}

1974). Given that learning cue validities can be time consuming and inaccurate (Gluck \& Bower, 1988; Goodie \& Crooks, 2004; Himmelfarb, 1970; Peterson \& Pitz, 1985), we propose that people might use a cue's fluency, or the ease with which it is processed, as an additional basis for weighting cues. A fluency-based account of cue weighting does not require decision-makers to know objective cue validities. Instead, this explanation highlights a rather simple tendency: placing more weight on information that feels easy to process.

\subsection{Fluency and its Applications to Cue Weighting}

Many different factors can affect subjective feelings of ease, or fluency. For instance, perceptual fluency refers to the ease with which a stimulus can be perceived (Jacoby, Woloshyn, \& Kelley, 1989). Retrieval fluency refers to the ease with which information can be brought to mind or recalled from memory (e.g., Tversky \& Kahneman, 1973). Other types of fluency include conceptual fluency (Whittlesea, 1993), linguistic fluency (Alter \& Oppenheimer, 2006; Oppenheimer, 2006; Whittlesea \& Leboe, 2000), and embodied fluency (Stepper \& Strack, 1993; for a review of different processes that engender fluency, see Alter \& Oppenheimer, 2007).

Broadly speaking, fluency can affect judgment and decision-making directly or indirectly. When fluency directly affects judgments, the ease of processing itself acts as a piece of information that can be weighted and applied to a judgment. A prominent example of direct fluency effects is the availability heuristic (Tversky \& Kahneman, 1973, 1974), by which people judge easy-to-recall events to be more likely to occur in the future. Jacoby and Dallas 
(1981) have also shown how processing fluency might directly affect judgments, where people are more likely to say that they have seen easy-to-read words before in the context of a recognition task.

When fluency indirectly affects judgments, the ease of processing might determine which cues people attend to, and the strategies they use to weight and integrate the information contained in those cues. That is, fluency is not itself used as a cue for judgment, but instead manipulates which strategies or cues are used to confront a task. These strategies and other pieces of information in turn affect the final judgment. For example, Alter, Oppenheimer, Epley, and Eyre (2007) found that participants who solved math and reasoning tasks were more likely to engage in systematic processing when they experienced disfluency, and heuristic processing when they experienced fluency. Note that the feelings of ease were not used as pieces of information that could be used to solve the math problems. Instead, fluency affected which strategies were used to answer the questions and these strategies in turn determined the final answers. This demonstrates how fluency might indirectly affect judgment by first leading people to adopt a particular strategy.

In this paper, we aim to extend the literature on how fluency might indirectly affect judgment. Specifically, we demonstrate that people's cue-weighting strategies depend on a cue's fluency. That is, people weight fluent cues more heavily than disfluent cues. This prediction stems from a review of the literature on heuristics which shows that people often heavily weight cues that are easier to access (Shah \& Oppenheimer, in press). For instance, people might use audience responsiveness as a cue for speaker/message persuasiveness (Axsom, Yates, $\&$ Chaiken, 1987), feelings of outrage as a cue for deciding punishment (Kahneman \& Frederick, 2002), brand name as a cue for product quality (Maheswaran, Mackie, \& Chaiken, 1992), or attractiveness as a cue for familiarity (Monin, 2003). Proposed heuristics such as these quietly converge on an underlying domain-general mechanism in which cues that are easy to process or retrieve are weighted more heavily than other cues. However, to date this mechanism has not been studied directly.

There are also other reasons that fluency emerges as a strong candidate for a mechanism underlying cueweighting. Such a mechanism makes sense given limited cognitive resources (Simon, 1955). Using cues which are easy to access will reduce the demands placed on decision-makers. Additionally, decision-makers might use fluency as the basis for cue weighting because they believe that more fluent cues are more valid. For example, Reber and Schwarz (1999) showed that more fluent statements are judged to be truer than less fluent statements. Additionally, Unkelbach $(2006,2007)$ has shown that the more strongly fluency is correlated with truth in a particular experimental setting, the stronger the inferred relationship between fluency and truth. As such, fluency could be used as a basis for cue weighting because fluency and objective validity might actually be correlated in the real world. It may then be the case that more fluent cues are subjectively seen as more valid, allowing for decision-makers to use fluency as a basis for cue weighting when objective validity information is absent or difficult to access.

To understand how fluency and validity might be correlated, consider a basic associative learning framework. From this framework, we can surmise that, whenever a decision-maker experiences an event in the presence of a cue, there is some probability that an association between the event and the cue will be learned. Cues that are seen more often in the presence of the event will become more strongly associated with the event. When decisionmakers later make judgments about the event, cues will be differentially primed based on their associations with the event. That is, cues that are more strongly associated with the event, will be easier to access when making judgments about the event. Since one consequence of priming is an increase in fluency (Stark \& McClelland, 2000), there will be a reliable relationship between the fluency of a cue and the objective validity of the cue.

Although cue fluency and objective validity might be correlated, they are separable concepts. For example, making a stimulus easier to perceive by increasing figureground contrast need not affect how accurately this stimulus predicts an outcome. Consequently, fluency can be manipulated separately from objective cue validity, allowing us to identify its unique contributions to cueweighting behavior.

In the remainder of this paper we describe three studies in which fluency affects how people weight cues. In all three studies fluency was manipulated independently of validity. In Experiment 1, participants priced an MP3 player based on product specifications and a negative consumer review. The consumer review was presented in either fluent or disfluent font. Participants weighted the consumer review more heavily if it was presented in a more fluent font. In Experiment 2, participants rated political lobbying firms based on brief recommendations from reviewers. These recommendations were a part of graphic logos that were either fluent or disfluent. As expected, participants weighted information from fluent images more than from disfluent images. In Experiment 3, participants made ratings about stocks based on evaluations from brokerage firms that were either fluently or disfluently named. Participants weighted information from the fluently named brokerage firms more heavily. These three studies demonstrate that perceptual fluency and linguistic fluency both affect how people select and weight cues when making judgments. 


\section{Experiment 1}

In this study participants indicated how much they believed an MP3 player should cost based on two types of information: product specifications and a consumer review. Holding the fluency and content of the product specifications constant, we used easy-to-read and hardto-read fonts to manipulate the perceptual fluency of the consumer review. We expected that participants would weight the consumer review more heavily when it was fluent than when it was disfluent.

\subsection{Method}

\subsubsection{Participants}

One hundred eight people participated in the study. Twenty-eight participants completed the survey as part of a larger packet of questionnaires and received $\$ 10$ compensation. The remaining eighty participants were recruited from a train station in Princeton Junction, NJ.

\subsubsection{Design, stimuli, and procedure}

Participants were asked to rate how much they thought an MP3 player should cost (between $\$ 0$ and $\$ 300$ ) based on its specifications and a negative consumer review. The specifications, consumer review, and rating scale were all contained on the same page. The specifications were held constant, while the fluency of the consumer review was manipulated. The fluent consumer review was presented in black 12-pt Times New Roman font, while the disfluent consumer review was presented in grey 12-pt italicized Monotype Corsiva font (see Appendix A for a scaled version of the survey and font types).

Our purpose behind using a negative consumer review was twofold. First, we wanted to demonstrate that fluency was not directly affecting judgment, but that it was instead affecting how people weighted information before making a judgment. Second, we wanted to rule out the possibility that mood states were also influencing judgments. If fluency were directly affecting people's judgment, then we might expect mood states to mediate this effect (Winkielman, Schwarz, Reber, \& Fazendeiro , 2003; Zajonc, 1968). Both direct-influence and affective accounts predict that participants in this study would price the MP3 player lower in the disfluent condition than in the fluent condition; either because the disfluency is a direct cue for a lack of quality, or because disfluency creates negative affect which in turns leads to lower evaluations. However, we predict that if fluency indirectly affects judgment, participants will price the MP3 player higher in the disfluent condition because they will place less weight on the negative disfluent cue. ${ }^{1}$

\footnotetext{
${ }^{1}$ We are using the term "cue" rather loosely here. We define any
}

Half of the participants were randomly assigned to the disfluent consumer review condition, and half were randomly assigned to the fluent consumer review condition. The data from nine participants were excluded from the analysis because these participants either did not answer the question or they provided a price that was out of the specified price range.

\subsection{Results and discussion}

As expected, participants who read a fluent consumer review priced the MP3 player significantly lower $(M=$ $\$ 126.29, S E=11.70)$ than did participants who read a disfluent consumer review $(M=\$ 162.06, S E=11.90, t(97)$ $=2.14, p=.035$, Cohen's $d=.43$ ).

The results suggest that participants weighted fluent information more heavily than disfluent information. That is, fluency indirectly affected the judgments. This study also enables us to rule out the possibility that mood changes are driving these effects. If the disfluency were leading to negative affect and this mood change were altering the judgments, then disfluent consumer reviews should have led to lower evaluations of the MP3 player's price. This was not the case. Instead, participants priced the MP3 player higher in the disfluent condition, indicating that they were not weighting the negative consumer review as heavily. This alternative explanation therefore appears weaker, and the findings more clearly support the hypothesis that fluency affects how people weight cues.

This study did suffer from a few limitations, however. First, the consumer review did not provide a concise evaluation that can be represented on a clear scale (such as number of gigabytes or hours of battery life). Given the length of the review, it is possible that participants in the disfluent condition simply did not read the entire consumer review. Additionally, fluency was manipulated only between subjects and so it remains unclear as to whether participants would still weight fluent cues more heavily if they were exposed to both fluent and disfluent cues. To address these limitations, we used withinsubjects fluency manipulations in the remaining studies and used cues containing more concise information.

\section{Experiment 2}

In Experiment 2, participants were given several pieces of background information about a fictional lobbying group,

information that should be weighted in the decision as a cue. The consumer review contains several pieces of information, each of which might be individually referred to as cues. For instance, it contains information about the durability of the product and specific problems. We recognize that the consumer review contains several cues, but expect that all of them will be weighted less heavily when the review is disfluent than when it is fluent. 
as well as information from two fabricated ratings indices for evaluating lobbying groups. These ratings indices were presented as images, and fluency was manipulated by darkening and blurring the images. Participants predicted the quality of the lobbying groups, and weighted a fluently presented rating more heavily than a disfluently presented rating.

\subsection{Method}

\subsubsection{Participants}

One hundred thirty-nine Princeton University students and staff members were recruited from the campus center. They completed the survey as part of a packet of questionnaires and received $\$ 12$ compensation.

\subsubsection{Design, stimuli, and procedure}

Participants were given a brief description of how some political lobbying groups operate. They were then asked to rate the quality of one fictional lobbying firm. Participants were given background information for the lobbying firm (location, number of employees, and the percentage of employees who once worked in Congress), which was held constant across all conditions.

Participants were also told that the industry often relies on two rating indices when evaluating the strength of lobbying groups - the Rose and Stone indices. The Rose index gave numeric ratings and the Stone index gave semantic ratings. We used different modalities for ratings so as to discourage the simple strategy of averaging the ratings from each index. For each index, we constructed graphical logos which contained the rating scores given for the lobbying group. Participants were told that these indices typically agree in their evaluations, but sometimes disagree. The indices shown to participants disagreed; when one index gave the lobbying firm a high rating, the other index gave a low rating. The assignment of high and low ratings to each index was counterbalanced across participants.

We manipulated the fluency of the cues (i.e., rating index logos) such that in the disfluent condition the logos were darkened and slightly blurred, while in the fluent condition the logos were left light and focused (see Figure 1 for sample stimuli). Half of the participants saw logos such that the fluent cue gave a high rating, and half of the participants saw logos such that the disfluent cue gave a high rating. The data from 11 participants were excluded from the analysis because these participants did not answer all of the questions.

Participants were asked to rate the quality of the lobbying groups along three dimensions. First, participants were asked to rate how good the lobbying firm was on a scale of 1 (low) to 100 (high). Second, participants predicted how much money a lobbying firm would win from Congress if it was hired to win \$2 million. Third, participants rated how likely they would be to recommend this lobbying firm on a scale of 1 (not likely at all) to 6 (very likely). The z-scores for these ratings were calculated, and the three ratings proved to be internally consistent (Cronbach's alpha = .67). As such, the z-scores were averaged for each response to form a composite quality rating. If fluency affects cue weighting, then we would expect participants to rate firms as better when the fluent cue gives a high rating, compared to when the disfluent cue gives a high rating.

\subsection{Results and discussion}

When analyzing the data, we combined cases where the fluent cue gave a high rating, regardless of whether that cue was the Rose or Stone index. ${ }^{2}$ Similarly, we combined cases where the disfluent cue gave a high rating. As predicted, participants who saw fluent cues give high ratings rated the lobbying firms as significantly better $(M$ $=.15, S E=.09)$ than did participants who saw disfluent cues give high ratings $(M=-.15, S E=.10, t(126)=2.15$, $p=.03$, Cohen's $d=.39$ ).

These results provide additional support for the hypothesis that fluency indirectly affects judgment, with participants placing more weight on fluent cues compared to disfluent cues. Moreover, this experiment replicates the above findings with a within-subjects fluency manipulation, indicating that people will weight fluent cues more heavily even when they are exposed to multiple pieces of information, some of which are more fluent than others. Additionally, given that the information presented was extremely concise, it is unlikely that participants were simply not reading through or retaining the information presented.

In these two studies, we manipulated the ease with which information could be read without manipulating objective cue validity. Although objective validity is not affected by fluency, participants' subjective impressions of validity may have been affected by cue fluency. A quick test of this hypothesis suggests that fluency does indeed affect subjective impressions of validity. In a separate session, seventy-five participants completed a similar task as that described above. However, instead of rating the lobbying group, participants rated how accurate they thought the different rating firms were. Responding on a four-point scale, these participants believed firms with

\footnotetext{
${ }^{2}$ It should be noted that the Rose index always conveyed numeric information, while the Stone index always conveyed semantic information. Numeric information was weighted somewhat more heavily than semantic information overall. However, for each index the expected trend is evident: fluent information is weighted more heavily than disfluent information.
} 

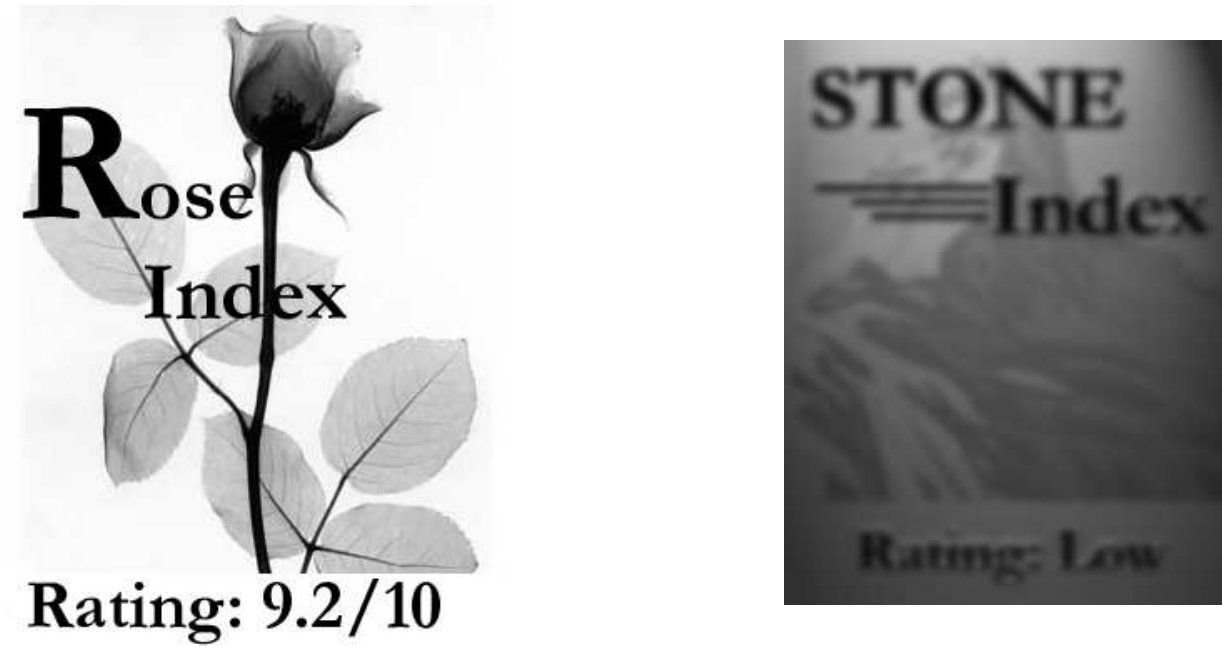

Figure 1: Stimuli from Experiment 2 with the Rose logo presented fluently the Stone logo presented disfluently.

fluent $\log$ os to be more accurate $(M=3.32, S E=.09)$ than firms with disfluent logos $(M=2.30, S E=.14)$, accounting for unequal variances $(t(61.83)=5.93, p<.001$, Cohen's $d=1.38$ ). This provides evidence for the hypothesis that fluency may affect cue weighting by altering subjective impressions of cue validity.

Although these studies provide strong support for our hypothesis, they are still limited to perceptual fluency. To make inferences about subjective feelings of ease in general, it is necessary to investigate other fluency manipulations as well. In Experiment 3, we find support for our hypothesis from the domain of linguistic fluency, or the ease with which words can be pronounced.

\section{Experiment 3}

In this experiment, we investigated whether linguistic fluency might also affect cue weighting (for examples of linguistic fluency manipulations, see Alter \& Oppenheimer, 2006; Whittlesea \& Leboe, 2000). Participants rated stocks along several dimensions based on a series of cues. Target cues were either easy to pronounce or difficult to pronounce. Ultimately, easier to pronounce cues were weighted more heavily than more difficult to pronounce cues.

\subsection{Method}

\subsubsection{Participants}

One hundred forty-four members of the Princeton University community (median age $=21$ ) were recruited to complete the survey as part of an online questionnaire.
They were compensated with entry into a lottery to win one of several gift certificates.

\subsubsection{Design, stimuli, and procedure}

Participants read about a fictitious stock with a series of cues that could be used for rating the stock's growth potential. Three of the cues were uninformative decoys (location of headquarters, date that the stock went public, and number of employees) and were held constant. The remaining two cues were stock evaluations from two fictional brokerage firms.

The fluency of the brokerage firms' names was manipulated within-subjects. However, we believed that it might be possible for participants to think that disfluent names reflected negatively on a company if there was no reason for the name to be disfluent. For example, participants might believe that a hard to pronounce name was indicative of a poorly managed brokerage firm. Such confounds would make it difficult for us to isolate the effects of fluency. To avoid this problem, we told participants that they were rating Turkish stocks and that the brokerage firms were also Turkish. Given that these were foreign brokerage firms, participants could naturally expect some names to be difficult to pronounce.

To manipulate the fluency of the brokerage firms' names, we used Turkish names that were shown in a pretest to be highly disfluent or highly fluent. In the pretest, a separate pool of 31 participants were asked to rate how easy it would be to pronounce different names on a scale of 1 (very easy) to 10 (very difficult). From 175 tested names, we selected the eight most fluent $(M=$ 2.74, $S E=.03)$ and eight most disfluent $(M=6.87, S E=$ .15 ), making up eight pairs of fluent/disfluent names (see Appendix B for a list of the names used). 
To make direct comparisons between the two cues more difficult, the information from the cues was given in different formats: letter grades versus linguistic descriptions. Cue fluency and information format were counterbalanced. A description of the cues was provided for the participants. The two key cues were described as "the [brokerage firm name] rating evaluates each stock along a verbal scale, ranging from 'Very Low' (poor stock choice) to 'Very High' (good stock choice)" and "the [brokerage firm name] rating gives letter grades to each stock, ranging from D- (poor stock choice) to A+ (good stock choice)." The spatial position, value, and modality of the fluent and disfluent cues (i.e., brokerage firm ratings) were fully counterbalanced.

Participants were told that the brokerage firms typically agree, although they might sometimes disagree. For the stock the participants were asked to review, one brokerage firm gave a positive evaluation, while the other brokerage firm gave a negative evaluation. Participants rated each stock on three factors. First, participants were asked to rate how well the stock would perform on a scale of 1 (low) to 100 (high). Second, participants predicted how much an initial investment of $\$ 1000$ in shares of the company would be worth in one year. Third, participants rated how likely they would be to recommend this stock to Turkish investors on a scale of 1 (not likely at all) to 6 (very likely). The data from three participants were excluded from the analyses because two participants indicated that they understood Turkish (thus the names would not be differentially fluent to them) and one participant did not answer all of the questions.

\subsection{Results and discussion}

We conducted our analyses by collapsing across all fluent-disfluent pairs of brokerage firms. Participants who saw fluently named brokerage firms give high ratings indicated that the stocks would perform better $(M$ $=58.08, S E=1.87)$ than did participants who saw disfluently named brokerage firms give high ratings $(M=$ 52.27, $S E=2.13, t(139)=2.06, p=.04$, Cohen's $d=.35)$. Furthermore, participants who saw fluent cues give high ratings indicated they were more likely to recommend the stock to Turkish investors $(M=3.49, S E=.11)$ than did participants who saw disfluent cues give high ratings $(M$ $=2.95, S E=.12, t(139)=3.36, p=.001$, Cohen's $d=.57)$. Finally, participants who saw fluent cues give high ratings also predicted that their initial investment of $\$ 1000$ would be worth more in one year $(M=\$ 1135, S E=58.26)$ than did participants who saw disfluent cues give high ratings $(M=\$ 1100, S E=38.79)$, but not significantly so $(t(139)$ $=.49, p=.62)$. The lack of a significant effect in the monetary evaluations may stem from the high variability that resulted from using an open-ended scale.
These data therefore suggest that people weight information from linguistically fluent sources more heavily than information from disfluent sources when evaluating stocks based on the recommendations of brokerage firms. Consequently, it becomes clear that a cue's linguistic fluency, in addition to its perceptual fluency, affects how heavily the cue is weighted. That is, convergent evidence from multiple fluency manipulations suggests that fluency operates as a domain-general basis for cue weighting.

\section{General discussion}

Although normative models propose that decisionmakers must weight cues by their objective validity, it has been shown time and again that this is a difficult undertaking (Evans, Clibbens, Cattani, Harris, \& Dennis, 2003, Goodie \& Crooks, 2004; Permut, 1973; Peterson \& Pitz, 1985; Tversky \& Kahneman, 1974). In this paper, we have proposed that people will often weight information according to the ease with which it can be processed.

This application of fluency theory builds upon previous fluency research, but emphasizes an indirect effect of fluency. Research on people's experiences of fluency typically investigates how fluency itself serves as a cue. Tversky and Kahneman's (1973, 1974) availability heuristic, for instance, indicates how events that come easily to mind will be judged as more likely to occur again in the future. Although we do not dispute that fluency is often used as a direct cue for judgment, our theory suggests that fluency may also affect judgments indirectly. In this sense, fluency can serve as a basis for deciding which cues will be weighted most heavily.

We found support for this application of fluency in several domains. Experiments 1 and 2 showed how perceptual fluency can affect cue weighting in both betweensubjects and within-subjects manipulations. Experiment 3 extended these findings to the domain of linguistic fluency. We were also able to rule out the possibility that mood changes would mediate the effect of fluency by using within-subject manipulations (Experiments 2 and 3 ) and using a design in which our hypothesis diverged from a mood-based account of fluency effects (Experiment 1). Although any individual fluency manipulation might also alter other variables, by using convergent evidence from different fluency manipulations we can more strongly state that it is indeed fluency that is affecting how people are deciding to weight information.

In each of these studies, fluency was manipulated independently of objective cue validity. However, we did find preliminary support for the idea that fluency affects decision-makers' subjective impressions of cue validity. Such findings suggest that assessing the fluency of cues 
may allow decision-makers to form impressions of cue validity before integrating the information from the task environment. In these studies, it might also be possible that participants attended more to fluent information than disfluent information, and that this attentional difference was the source of the cue-weighting effects. Although this account is consistent with the basic hypothesis that fluent information will affect judgments more than disfluent information, it would be useful to further demonstrate that people pay equal attention to fluent and disfluent information, but weight fluent information more heavily. Additionally, a larger set of judgments per participant would make it possible to obtain actual beta weights for each cue to provide stronger evidence for the hypothesis.

On a related note, it might be possible that people simply select accessible or fluent information until they feel they have enough information and then disregard the rest of the information. That is, fluency might determine whether information is used, but not how that information is differentially weighted. ${ }^{3}$ This noticeably differs from our hypothesis that disfluent cues will still be weighted, only less heavily. This is a possibility; however, it appears that participants in the studies above were weighting information from disfluent sources as well. Consider the data from the third study - for both closed-scale responses (likelihood of recommending the stock and stock performance), participants' mean ratings were clustered around the midpoint of the scales. This suggests that both the extreme positive cue and the extreme negative cue were affecting their judgments, but that the fluent cue was weighed slightly more heavily. Although this provides some post-hoc evidence for subtle cue weighting, actual regression coefficients would support the case more strongly.

It may also be argued that if the participants had a chance to learn cue validities then objective validity would have also affected cue weighting. We are sympathetic to this point. We do not argue that fluency is the sole mechanism by which people weight cues. Instead, we argue that fluency affects cue weighting when validity information is not present and that fluency may itself be a proxy for cue validity. However, the fact that we did not manipulate cue validity provides questions for further research into whether fluency will affect cue weighting when objective validity information is known.

Some may also note similarities between the hypothesis that ease of processing affects cue weighting and the evaluability hypothesis (Hsee, Loewenstein, Blound, \& Bazerman, 1999). The evaluability hypothesis describes how attributes or cues can vary in how easy they are to assess in joint- versus separate-evaluation tasks. Attributes that are easy to evaluate independently will carry

\footnotetext{
${ }^{3}$ We thank an anonymous reviewer for drawing our attention to this useful distinction.
}

more weight in separate evaluation. However, attributes that are hard to evaluate independently might carry more weight in joint evaluation (if juxtaposing two options makes it easier to compare and evaluate the options along this attribute). Although the fluency-based account and the evaluability-based account of cue weighting are similar in their emphasis on ease of processing, the two theories diverge in describing how cues become easy to process. The fluency-based theory points to perceptual and retrieval ease, while the evaluability hypothesis focuses on the ease of mapping attribute values onto an evaluative scale. Although these theories differ, they are by no means mutually exclusive and instead complement each other by emphasizing ways in which decision-makers attempt to reduce cognitive effort.

Indeed, one of the original rationales for developing a fluency-based account of cue weighting was the belief that people may use fluent cues in order to save cognitive effort. This leads to a prediction for future studies. If people are indeed using easier to access information because this information provides a shortcut when confronting a decision task, then this strategy ought to be used more often when participants are placed under cognitive load.

Another rationale for our hypothesis was the belief that fluency was an ecologically valid proxy for cue validity because of associative learning. Future research should investigate the extent to which increases in cue validity actually lead to increases in fluency. If manipulating the fluency of a cue can alter its perceived validity, then altering the objective validity of a cue might also alter its fluency.

Although there is still much to be learned about how fluency affects cue weighting, the evidence described above opens the door to such research by demonstrating the effect of fluency on cue weighting when objective validities are unknown. These findings show how fluency can affect numeric judgments indirectly, building on the numerous studies where fluency affects judgment directly. Furthermore, the idea that fluency can affect cue weighting provides further insight into how decisionmakers often seek to reduce cognitive effort. And it ultimately appears that the extent to which we use information when making judgments may depend on a very basic feature: how easy it is to process.

\section{References}

Alter, A. L., \& Oppenheimer, D. M. (2006). Predicting short-term stock fluctuations by using processing fluency. Proceedings of the National Academy of Sciences, 103, 9369-9372.

Alter, A. L., Oppenheimer, D. M., Epley, N., \& Eyre, R. N. (2007). Overcoming intuition: Metacognitive diffi- 
culty activates analytic reasoning. Journal of Experimental Psychology: General, 136, 569-576.

Alter, A. L., \& Oppenheimer, D. M. (2007). Uniting the tribes of fluency to form a metacognitive nation. Manuscript under review.

Axsom, D., Yates, S., \& Chaiken, S. (1987). Audience response as a heuristic cue in persuasion. Journal of Personality and Social Psychology, 53, 30-40.

Evans, J. St. B. T., Clibbens, J., Cattani, A., Harris, A., \& Dennis, I. (2003). Explicit and implicit processes in multicue judgment. Memory and Cognition, 31, 608618.

Gluck, M. A., \& Bower, G. H. (1988). Evaluating an adaptive network of human learning. Journal of Memory and Language, 27, 166-195.

Goodie, A. S., \& Crooks, C. L. (2004). Time pressure effect on performance in a base-rate task. Journal of General Psychology, 131, 18-28.

Himmelfarb, S. (1970). Effects of cue validity differences in weighting information. Journal of Mathematical Psychology, 7, 531-539.

Hsee, C. K, Loewenstein, G. F., Blount, S. \& Bazerman, M. H. (1999). Preference reversals between joint and separate evaluation of options: A review and theoretical analysis. Psychological Bulletin, 125, 576-590.

Jacoby, L. L., \& Dallas, M. (1981). On the relationship between autobiographical memory and perceptual learning. Journal of Experimental Psychology: General, 110, 306-340.

Jacoby, L. L., Woloshyn, V., \& Kelley, C. (1989). Becoming famous without being recognized: Unconscious influences of memory produced by divided attention. Journal of Experimental Psychology: General, 118, 115-125.

Kahneman, D., \& Frederick, S. (2002). Representativeness revisited: Attribute substitution in intuitive judgment. In T. Gilovich, D. Griffin, \& D. Kahneman (Eds.), Heuristics and biases: The psychology of intuitive judgment (pp. 49-81). New York: Cambridge University Press.

Maheswaran, D., Mackie, D. M., Chaiken, S. (1992). Brand name as a heuristic cue: The effects of task importance and expectancy confirmation on consumer judgments. Journal of Consumer Psychology, 1, 317336.

Monin, B. (2003). The warm glow heuristic: When liking leads to familiarity. Journal of Personality and Social Psychology, 85, 1035-1048.

Oppenheimer, D. M. (2006). Consequences of erudite vernacular utilized irrespective of necessity: Problems with using long words needlessly. Applied Cognitive Psychology, 20, 139-156.

Payne, J. W., Bettman, J. R., \& Johnson, E. J. (1993). The adaptive decision maker. New York: Cambridge
University Press.

Permut, S. E. (1973). Cue utilization patterns in studentfaculty evaluation. Journal of Psychology: Interdisciplinary and Applied, 83, 41-48.

Peterson, D. K., \& Pitz, G. F. (1985). Explicit cue weighting in a prediction task. Organizational Behavior and Human Decision Processes, 36, 289-304.

Reber, R., \& Schwarz, N. (1999). Effects of perceptual fluency on judgments of truth. Consciousness and Cognition: An International Journal, 8, 338-342.

Shah, A. K., \& Oppenheimer, D. M. (in press). Heuristics made easy: An effort-reduction framework. Psychological Bulletin.

Simon, H. A. (1955). A behavioral model of rational choice. The Quarterly Journal of Economics, 69, 99118.

Stark, C. E. L., \& McClelland, J. L. (2000). Repetition priming of words, pseudowords, and nonwords. Journal of Experimental Psychology: Learning, Memory, and Cognition, 26, 945-972.

Stepper, S., \& Strack, F. (1993). Proprioceptive determinants of emotional and nonemotional feelings. Journal of Personality and Social Psychology, 64, 211-220.

Tversky, A. (1969). Intransitivity of preferences. Psychological Review, 76, 31-48.

Tversky, A., \& Kahneman, D. (1973). Availability: A heuristic for judging frequency and probability. Cognitive Psychology, 5, 202-232.

Tversky, A., \& Kahneman, D. (1974). Judgment under uncertainty: Heuristics and biases. Science, 185, 1124-1131.

Unkelbach, C. (2006). The learned interpretation of cognitive fluency. Psychological Science, 17, 339-345.

Unkelbach, C. (2007). Reversing the truth effect: Learning the interpretation of processing fluency in judgments of truth. Journal of Experimental Psychology: Learning, Memory, and Cognition, 33, 219-230.

Whittlesea, B. W. A. (1993). Illusions of familiarity. Journal of Experimental Psychology: Learning, Memory, and Cognition, 19, 1235-1253.

Whittlesea, B. W. A., \& Leboe, J. P. (2000). The heuristic basis of remembering and classification: Fluency, generation, and resemblance. Journal of Experimental Psychology: General, 129, 84-106.

Winkielman, P., Schwarz, N., Reber, R., \& Fazendeiro, T. A. (2003). Cognitive and affective consequences of visual fluency: When seeing is easy on the mind. In L. M. Scott \& R. Batra (Eds.), Persuasive Imagery: A Consumer Response Perspective, (pp. 75-89). Mahwah, NJ: Lawrence Earlbaum Associates.

Zajonc, R. B. (1968). Attitudinal effects of mere exposure. Journal of Personality and Social Psychology, 9, $1-27$. 


\section{Appendix A: Survey used in Experi- Appendix B: Names used for broker- ment 1 age firms in Experiment 3}

Below is a description of a new MP3 player on the market. We have provided a few of the player's specs and one consumer review of the product. Once you have read through the information below, please answer the questions on this sheet. Thank you.

\section{MP3 Player: 20GB}

- Excellent sound quality and capacity (5,000 songs)

- Battery life: 12 hours

- Light weight

- Video capacity \& built in camera

\begin{tabular}{ll}
\hline Fluent & Disfluent \\
\hline Artan & Lasiea \\
Dermod & Siirt \\
Tatra & Aklale \\
Pera & Taahhut \\
Ferka & Emniyet \\
Kado & Dizayn \\
Alet & Luici \\
Boya & Sampiy \\
\hline
\end{tabular}

[Fluent Condition:]

Consumer Review:

It only lasted about 3 months. For whatever reason, I couldn't turn it on anymore. I then had to send it back, and I got another one after about a month. The new one has a busted directional pad that needs to be pushed in extremely hard for it to go up. It seems like a good product if it weren't so easy to break.

[Disfluent Condition:] 